\title{
Article
}

\section{Cell Cycle Regulation and DNA Damage Response Networks in Diffuse-and Intestinal-Type Gastric Cancer.}

\author{
Shihori Tanabe ${ }^{1, *}$, Sabina Quader ${ }^{2}$, Ryuichi Ono ${ }^{3}$, Horacio Cabral ${ }^{4}$, Kazuhiko Aoyagi ${ }^{5}$, Akihiko Hirose ${ }^{1}$, Hiroshi \\ Yokozaki ${ }^{6}$ and Hiroki Sasaki ${ }^{7}$
}

1 Division of Risk Assessment, Center for Biological Safety and Research, National Institute of Health Sciences, Kawasaki 210-9501, Japan; stanabe@nihs.go.jp (S.T.), hirose@nihs.go.jp (A.H.)

2 Innovation Center of NanoMedicine (iCONM), Kawasaki Institute of Industrial Promotion, Kawasaki 2100821, Japan; sabina-q@kawasaki-net.ne.jp

3 Division of Cellular and Molecular Toxicology, Center for Biological Safety and Research, National Institute of Health Sciences, Kawasaki 210-9501, Japan; onoryu@nihs.go.jp

4 Department of Bioengineering, Graduate School of Engineering, University of Tokyo, Tokyo 113-0033, Japan; horacio@bmw.t.u-tokyo.ac.jp

5 Department of Clinical Genomics, National Cancer Center Research Institute, Tokyo 104-0045, Japan; kaaoyagi@ncc.go.jp

6 Department of Pathology, Kobe University of Graduate School of Medicine, Kobe 650-0017, Japan; hyoko@med.kobe-u.ac.jp

7 Department of Translational Oncology, National Cancer Center Research Institute, Tokyo 104-0045, Japan; hksasaki@ncc.go.jp

* Correspondence: stanabe@nihs.go.jp; Tel.: +81-44-270-6686

Simple Summary: Epithelial-mesenchymal transition (EMT) is an important hallmark in drug resistance and cancer malignancy in cancer stem cells (CSCs). In this study, gene expression in diffuseand intestinal-type gastric cancer (GC) was investigated to reveal the precise mechanism of EMT. The pathways on cell cycle regulation and DNA damage response were found to be altered in diffuse- and intestinal-type GC. The findings of this study may provide broader insights into CSCs with new possibilities of the involvement of the cell cycle in EMT.

\begin{abstract}
Epithelial-mesenchymal transition (EMT) networks are essential in acquiring the drug resistance and cancer malignant features in cancer stem cells (CSCs). In this regard, gene expression profiles in diffuse- and intestinal-type gastric cancer (GC) have been analyzed to reveal the network pathways in EMT and CSCs, since the diffuse-type GC has much more mesenchymal features than intestinal-type GC that has the intestinal features. The study results revealed that the activation state of several canonical pathways related to cell cycle regulation was altered. The canonical pathway on Cell cycle: $\mathrm{G}_{1} / \mathrm{S}$ checkpoint regulation was activated in diffuse-type GC, and canonical pathways on Cell cycle control of chromosomal replication and Cyclins and cell cycle regulation were activated in intestinal-type GC. Canonical pathway related to Role of BRCA1 in DNA damage response was activated in intestinal-type GC, where BRCA1, which is related to G1/S phase transition was upregulated in intestinal-type GC. Several microRNAs (miRNAs), including mir-10, mir-17, mir-19, mir-194, mir-224, mir-25, mir-34, mir-451, and mir-605, were identified to have direct relationships of RNA-RNA interaction in Cell cycle: G1/S checkpoint regulation pathway. Additionally, cell cycle regulation may be altered in EMT conditions. The alterations in activation states of the pathways related to cell cycle regulation in diffuse- and intestinal-type GC would indicate the significance of cell cycle regulation in EMT.
\end{abstract}

Keywords: BRCA1; cancer stem cell; cell cycle; epithelial-mesenchymal transition; DNA damage response; gastric cancer; molecular network 


\section{Introduction}

In cancer stem cells (CSCs) in general, epithelial-mesenchymal transition (EMT) networks play an important role in acquiring the drug resistance and cancer malignant feature [1]. Alteration in gene expression of molecular network pathways results in the phenotypic changes. Diffuse-type GC has much more mesenchymal characteristics, which is an important feature of EMT, compared to intestinal-type GC. Accordingly, gene expression profiles have been analyzed for diffuse- and intestinal-type gastric cancer (GC) to reveal the network pathways in EMT and CSCs. Our previous findings identified several molecular networks and the related microRNAs (miRNAs) in intestinal- and diffuse-type GC $[2,3]$. Again, few recent studies have revealed the regulation of non-coding RNAs, including miRNAs in drug resistance and EMT in cancer [4-6]. It has been also been featured that the knockdown of circularNOP10, a circular RNA promoting tumor metastasis and EMT, decreased the numbers of cells in the $S$ phase and increased the number of cells in the $\mathrm{G}_{2} / \mathrm{M}$ phase [6].

The gene expression signature revealed that the ratio of $\mathrm{CDH} 2$ ( $\mathrm{N}$-cadherin) to $\mathrm{CDH} 1$ (E-cadherin) distinguishes the diffuse- and intestinal-type GC [7]. The gene expression and pathway analysis of CTNNB1 ( $\beta$-catenin) identified an important role of $\beta$-catenin signaling in the regulation of stem cell pluripotency and cancer signaling [8]. Although cell proliferation and cell cycle regulation are essential for identifying potential therapeutic targets in cancer, the precise mechanism of cell cycle regulation and EMT has not been fully revealed. This article focuses on the roles of cell cycle regulation pathways in diffuseand intestinal-type GC as cell cycle regulation may play a critical role in intestinal- and diffuse-type GC. Consequently, the mechanism of cancer drug resistance is highlighted through the involvement of the cell cycle in EMT and CSCs, which might reveal future therapeutic potentials.

\section{Materials and Methods}

\subsection{Data Analysis of Diffuse- and Intestinal-Type GC}

The RefSeq data of diffuse- and intestinal-type GC are publicly available in The Cancer Genome Atlas (TCGA) of The cBioPortal for Cancer Genomics database [9-11] in National Cancer Institute (NCI) Genomic Data Commons (GDC) Data Portal [12]. From the publicly available data of stomach adenocarcinoma in TCGA [10], intestinal- and diffusetype GC data, which are noted as chromosomal instability (CIN) $(n=223)$ and genomically stable (GS) ( $\mathrm{n}=50)$, respectively, in TCGA Research Network publication, were compared [11].

\subsection{Network Analysis}

Data of intestinal- and diffuse-type GC in TCGA cBioPortal Cancer Genomics were uploaded and analyzed through the use of Ingenuity Pathway Analysis (IPA) (QIAGEN Inc., Hilden, Germany) [13].

\subsection{Data Visualization}

The results of gene expression data of RefSeq and network analysis were visualized by Tableau software (https://www.tableau.com).

\subsection{Statistical Analysis}

The RefSeq data were analyzed by Student's t-test. Z-score in intestinal- and diffusetype GC samples were compared, and the difference was considered to be significant in $p$ value $<0.00001[2]$.

\section{Results}

3.1. Canonical Pathways in Diffuse- and Intestinal-Type GC 
Canonical pathways altered in diffuse- and intestinal-type GC are shown in Figure 1 and Table 1 . The 2815 IDs which are significantly different in diffuse- and intestinal-type GC were analyzed in network analysis, which identified 47 canonical pathways with absolute z-score $>1$ in the diffuse- and intestinal-type GC (Table 1). These canonical pathways included Cell cycle control of chromosomal replication, Cell cycle: G1/S checkpoint regulation, Cyclins and cell cycle regulation, Role of BRCA1 in DNA damage response, and Cell cycle: G2/M DNA damage checkpoint.

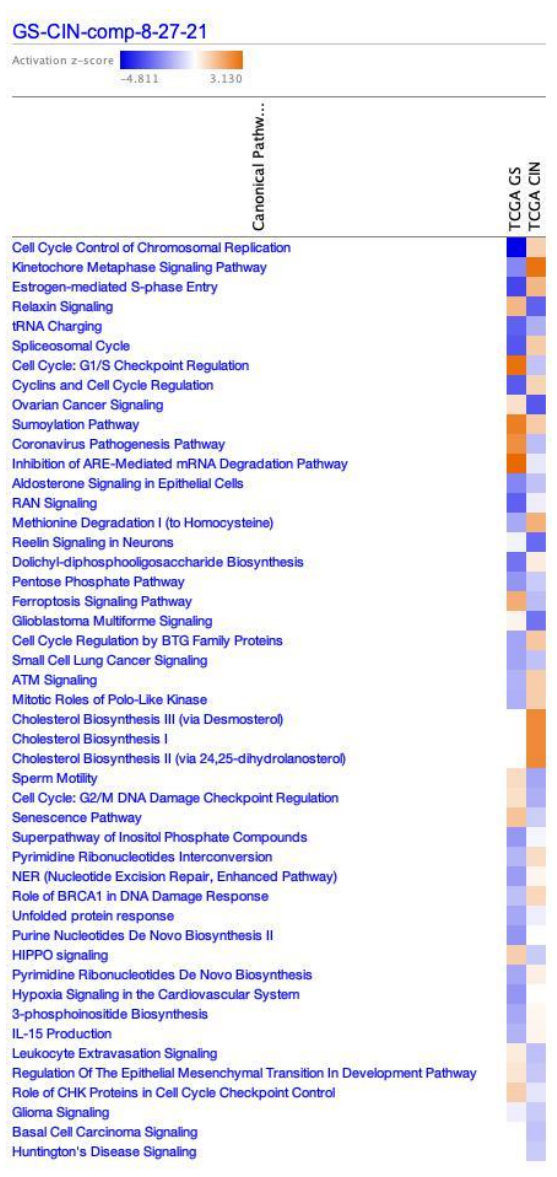

(a)

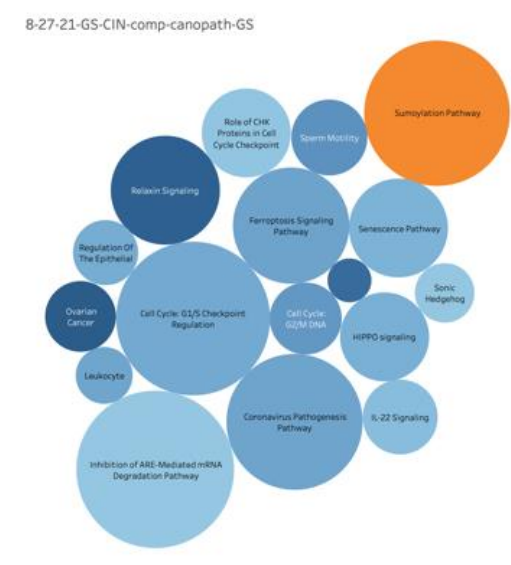

(b)

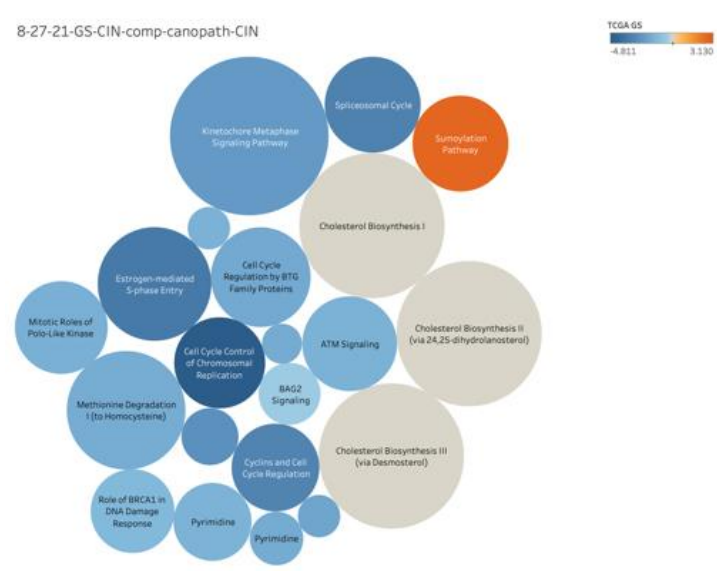

(c)

Figure 1. Canonical pathways altered in diffuse- and intestinal-type GC. (a) Canonical pathways with absolute z-score $>1$ in the diffuse- and intestinal-type GC are shown (TCGA GS; diffuse-type GC, TCGA CIN; intestinal-type GC); (b) Size shows the activation score in diffuse-type GC. Color indicates the activation score in intestinal-type GC.; (c) Size indicates the activation z-score in intestinal-type GC. Color indicates the activation z-score in intestinal-type GC.

Table 1. Canonical pathways altered in diffuse- and intestinal-type GC. The pathways are sorted by the order of the activation $\mathrm{z}-$ score.

\begin{tabular}{ccc}
\hline & Diffuse-type & Intestinal-type \\
Canonical Pathways & GC & GC \\
\hline Cell Cycle Control of Chromosomal Replication & -4.811 & 0.962 \\
\hline Kinetochore Metaphase Signaling Pathway & -2.271 & 2.92 \\
\hline Estrogen-mediated S-phase Entry & -3.5 & 1.5 \\
\hline Relaxin Signaling & 1.5 & -3 \\
\hline tRNA Charging & -3 & -1.5 \\
\hline Spliceosomal Cycle & -3.207 & 1.069 \\
\hline Cell Cycle: $\mathrm{G}_{1} /$ S Checkpoint Regulation & 2.982 & -1.147
\end{tabular}




\begin{tabular}{|c|c|c|}
\hline Cyclins and Cell Cycle Regulation & -3.13 & 0.894 \\
\hline Ovarian Cancer Signaling & 0.632 & -3.162 \\
\hline Sumoylation Pathway & 2.673 & 1.069 \\
\hline Coronavirus Pathogenesis Pathway & 2.335 & -1.257 \\
\hline Inhibition of ARE-Mediated mRNA Degradation Pathway & 3.13 & -0.447 \\
\hline Aldosterone Signaling in Epithelial Cells & -2.309 & -1.155 \\
\hline RAN Signaling & -3 & -0.333 \\
\hline Methionine Degradation I (to Homocysteine) & -1.633 & 1.633 \\
\hline Reelin Signaling in Neurons & -0.218 & -2.837 \\
\hline Dolichyl-diphosphooligosaccharide Biosynthesis & -2.646 & 0.378 \\
\hline Pentose Phosphate Pathway & -2 & -1 \\
\hline Ferroptosis Signaling Pathway & 1.706 & -1.279 \\
\hline Glioblastoma Multiforme Signaling & 0.243 & -2.668 \\
\hline Cell Cycle Regulation by BTG Family Proteins & -1.732 & 1.155 \\
\hline Small Cell Lung Cancer Signaling & -1.732 & -1.155 \\
\hline ATM Signaling & -1.46 & 1.043 \\
\hline Mitotic Roles of Polo-Like Kinase & -1.5 & 1 \\
\hline Cholesterol Biosynthesis III (via Desmosterol) & 0 & 2.449 \\
\hline Cholesterol Biosynthesis I & 0 & 2.449 \\
\hline Cholesterol Biosynthesis II (via 24,25-dihydrolanosterol) & 0 & 2.449 \\
\hline Sperm Motility & 0.728 & -1.698 \\
\hline Cell Cycle: $\mathrm{G}_{2} / \mathrm{M}$ DNA Damage Checkpoint Regulation & 0.655 & -1.528 \\
\hline Senescence Pathway & 1.234 & -0.926 \\
\hline Superpathway of Inositol Phosphate Compounds & -1.976 & -0.18 \\
\hline Pyrimidine Ribonucleotides Interconversion & -1.414 & 0.707 \\
\hline NER (Nucleotide Excision Repair, Enhanced Pathway) & -1.877 & 0.209 \\
\hline Role of BRCA1 in DNA Damage Response & -1.225 & 0.816 \\
\hline Unfolded protein response & -1.667 & -0.333 \\
\hline Purine Nucleotides De Novo Biosynthesis II & -2 & 0 \\
\hline HIPPO signaling & 1 & -1 \\
\hline Pyrimidine Ribonucleotides De Novo Biosynthesis & -1.667 & 0.333 \\
\hline Hypoxia Signaling in the Cardiovascular System & -2 & 0 \\
\hline 3-phosphoinositide Biosynthesis & -1.671 & 0.186 \\
\hline IL-15 Production & -1.46 & 0.209 \\
\hline Leukocyte Extravasation Signaling & 0.408 & -1.225 \\
\hline Regulation Of The Epithelial Mesenchymal Transition In Development Pathway & 0.535 & -1.069 \\
\hline Role of CHK Proteins in Cell Cycle Checkpoint Control & 1 & -0.5 \\
\hline Glioma Signaling & -0.333 & -1 \\
\hline Basal Cell Carcinoma Signaling & 0 & -1.155 \\
\hline Huntington's Disease Signaling & 0 & -1 \\
\hline
\end{tabular}

\subsection{Cell Cycle-Related Canonical Pathways in Diffuse- and Intestinal-Type GC}

3.2.1. Cell cycle control of chromosomal replication was activated in intestinal-type GC

Molecule activity predictor in IPA predicted the activation of Cell Cycle Control of Chromosomal Replication pathway in intestinal-type GC (Figure 2). Analysis on direct relationships of RNA-RNA interactions: microRNA targeting in Cell Cycle Control of Chromosomal Replication pathway revealed the relationships between miRNAs and the targeted molecules (Table 2). 


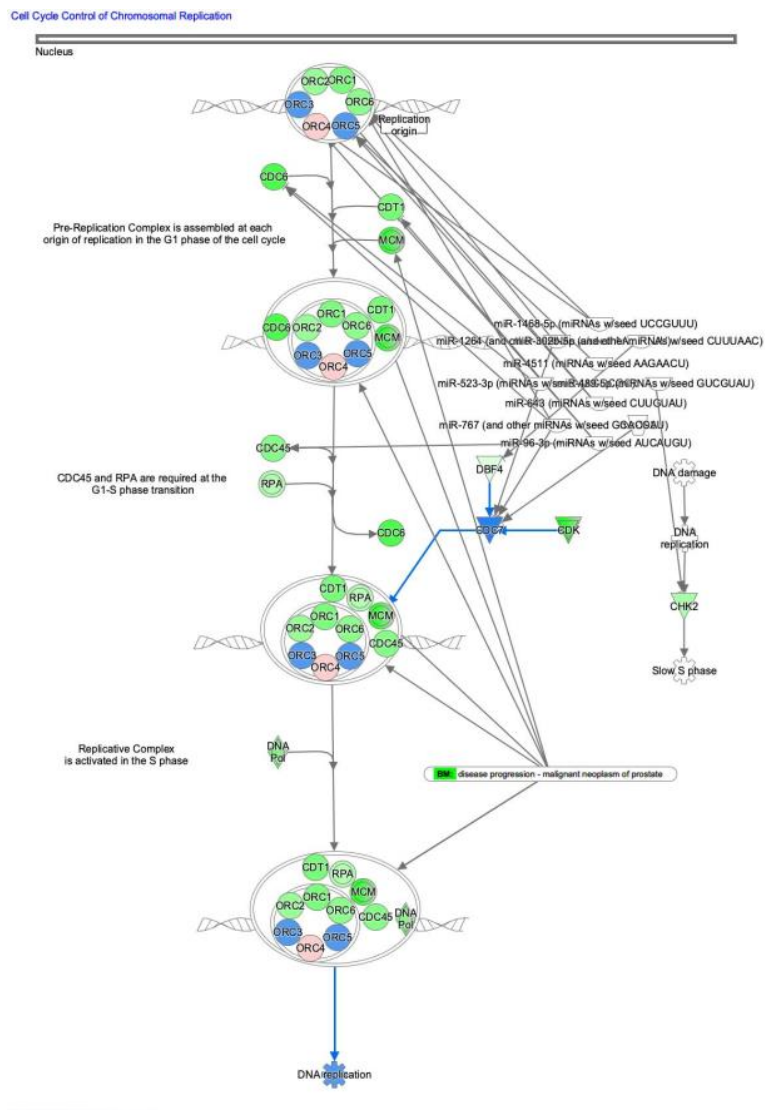

(a)

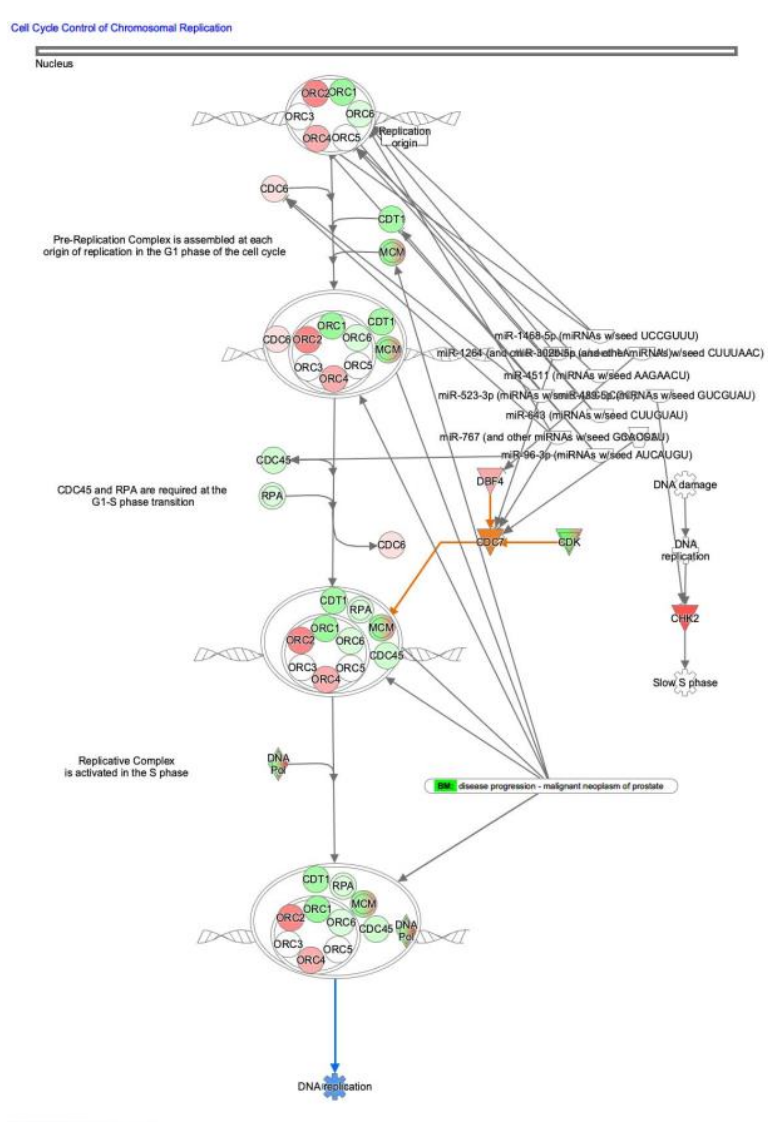

(b)

Figure 2. Cell cycle control of chromosomal replication was activated in intestinal-type GC. (a) Gene expression and pathway activity prediction in diffuse-type GC are shown; (b) Gene expression and pathway activity prediction in intestinal-type GC are shown. The genes of which expression was altered in diffuse- and intestinal-type GC are shown in pink (up-regulated) or green (down-regulated). Predicted activation or inhibition is shown in orange or blue, respectively.

Table 2. Direct relationships of miRNAs and targeted molecules in Cell cycle control of chromosomal replication.

\begin{tabular}{cc}
\hline From Molecule(s) & To Molecule(s) \\
\hline miR-1264 (and other miRNAs w/seed AA- & CDC7 \\
\cline { 2 - 2 } miR-1468-5p (miRNAs w/seed UCCGUUU) & ORC6 \\
\hline mir-192 & ORC4 \\
\hline miR-302b-5p (and other miRNAs w/seed & ORC6 \\
\hline miR-4511 (miRNAs w/seed AAGAACU) & CDC7 \\
\hline miR-489-5p (miRNAs w/seed GUCGUAU) & DBF4 \\
\hline miR-523-3p (miRNAs w/seed AACGCGC) & ORC5 \\
\hline miR-643 (miRNAs w/seed CUUGUAU) & CHK2 \\
\hline miR-767 (and other miRNAs w/seed GCAC- & CDC6 \\
\cline { 2 - 2 } CAU) & ORC2 \\
\hline & ORC5 \\
\hline miR-96-3p (miRNAs w/seed AUCAUGU) & CDC6 \\
\cline { 2 - 2 } & CDC7 \\
\hline
\end{tabular}




\subsubsection{Cell cycle: $\mathrm{G}_{1} / \mathrm{S}$ checkpoint regulation pathway was activated in diffuse-type GC}

Molecule activity predictor in IPA predicted the activation of Cell cycle: $G_{1} / S$ checkpoint regulation pathway in diffuse-type GC (Figure 3). In Cell cycle: G1/S checkpoint regulation pathway, DNA damage induces p53, which is expected to be activated in diffusetype GC. Analysis on direct relationships of RNA-RNA interaction in Cell cycle: G1/S checkpoint regulation pathway revealed the non-coding RNAs including 9 miRNAs and a biologic drug (MYC-targeting siRNA DCR-MYC) (Table 3).

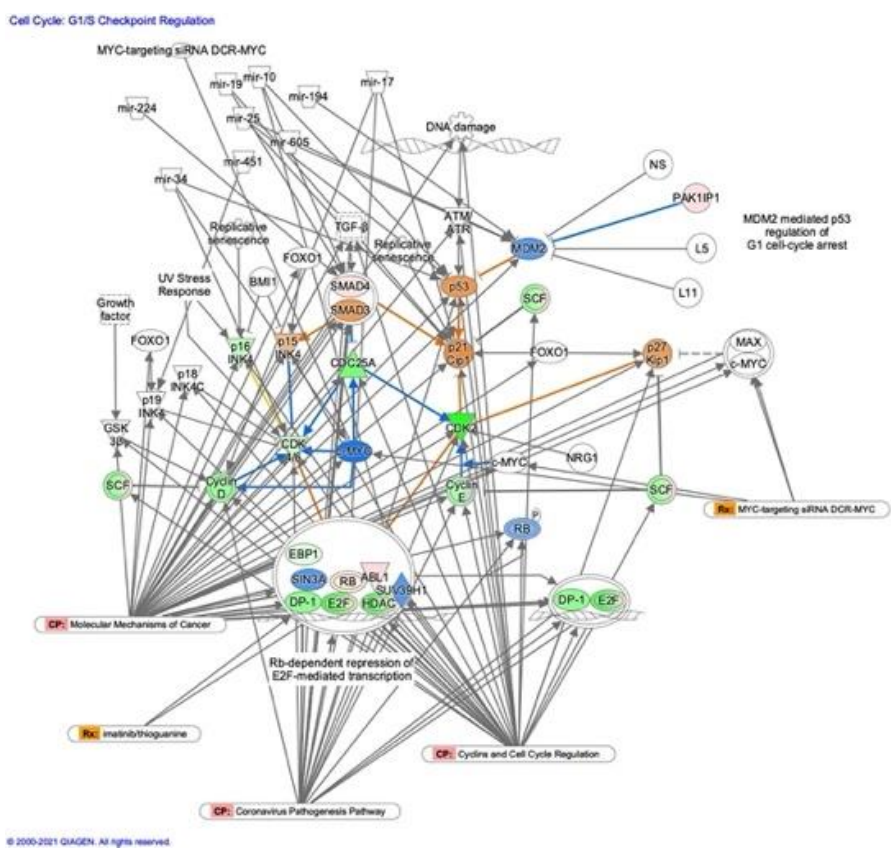

(a)

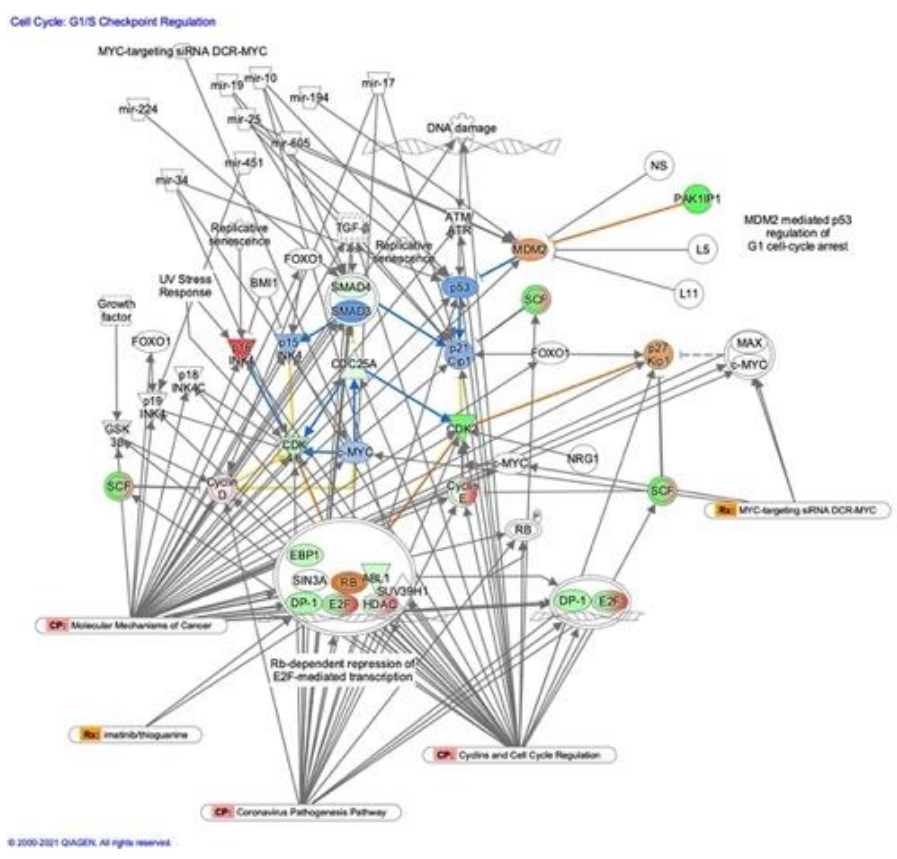

(b)

Figure 3. Cell cycle: G1/S checkpoint regulation pathway was activated in diffuse-type GC. (a) Gene expression and pathway activity prediction in diffuse-type GC are shown; (b) Gene expression and pathway activity prediction in intestinal-type GC are shown. The genes of which expression was altered in diffuse- and intestinal-type GC are shown in pink (up-regulated) or green (down-regulated). Predicted activation or inhibition is shown in orange or blue, respectively.

Table 3. Non-coding RNAs which have direct relationships in Cell cycle: G1/S checkpoint regulation pathway.

\begin{tabular}{|c|c|c|c|}
\hline Symbol & Entrez Gene Name & Location & Family \\
\hline mir-10 & microRNA 99a & \multirow{9}{*}{ Cytoplasm } & \multirow{9}{*}{ microRNA } \\
\hline mir-17 & microRNA 17 & & \\
\hline mir-19 & microRNA 19a & & \\
\hline mir-194 & microRNA 194-1 & & \\
\hline $\operatorname{mir}-224$ & microRNA 224 & & \\
\hline $\operatorname{mir}-25$ & microRNA 25 & & \\
\hline mir-34 & microRNA 34a & & \\
\hline $\operatorname{mir}-451$ & microRNA 451a & & \\
\hline mir-605 & microRNA 605 & & \\
\hline MYC-targeting siRNA DCR-MYC & & Other & biologic drug \\
\hline
\end{tabular}


3.2.3. Cyclins and cell cycle regulation pathway was activated in intestinal-type GC

Molecule activity predictor in IPA predicted the activation of Cyclins and cell cycle regulation pathway in intestinal-type GC (Figure 4). Analysis on direct relationships of RNA-RNA interactions: microRNA targeting in Cyclins and cell cycle regulation pathway revealed the relationships between miRNAs and the targeted molecules (Table 4).

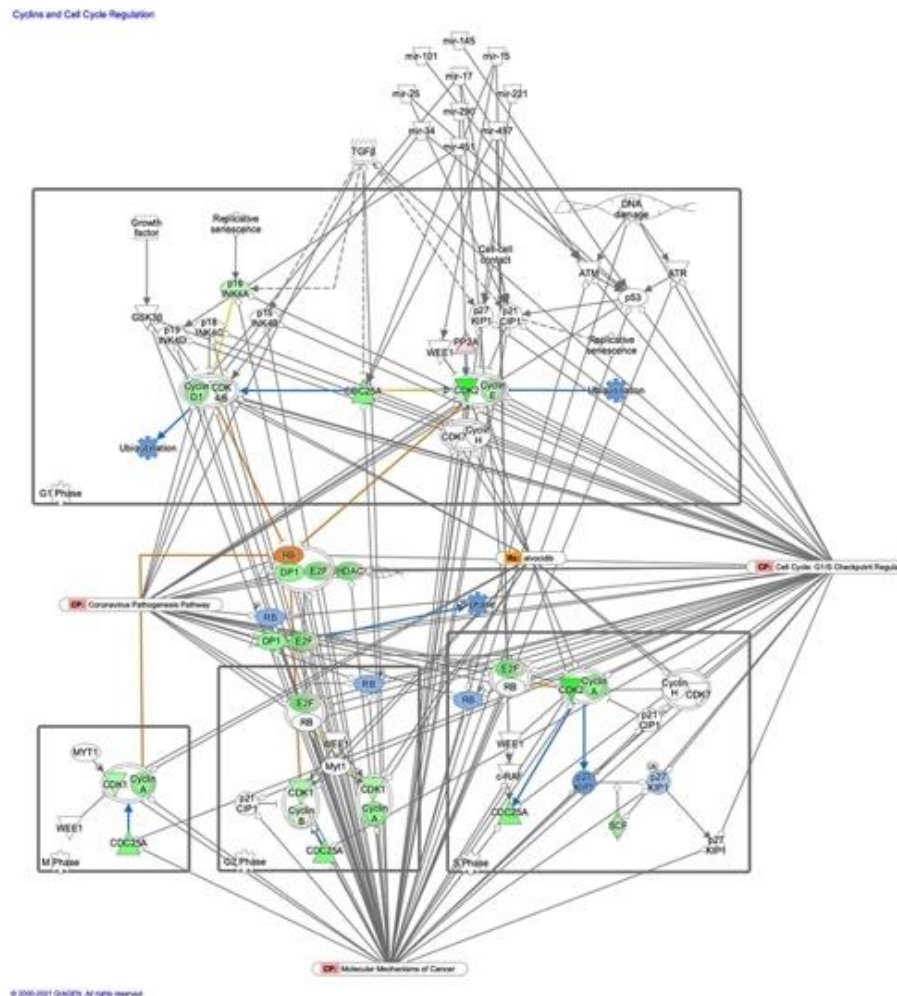

(a)

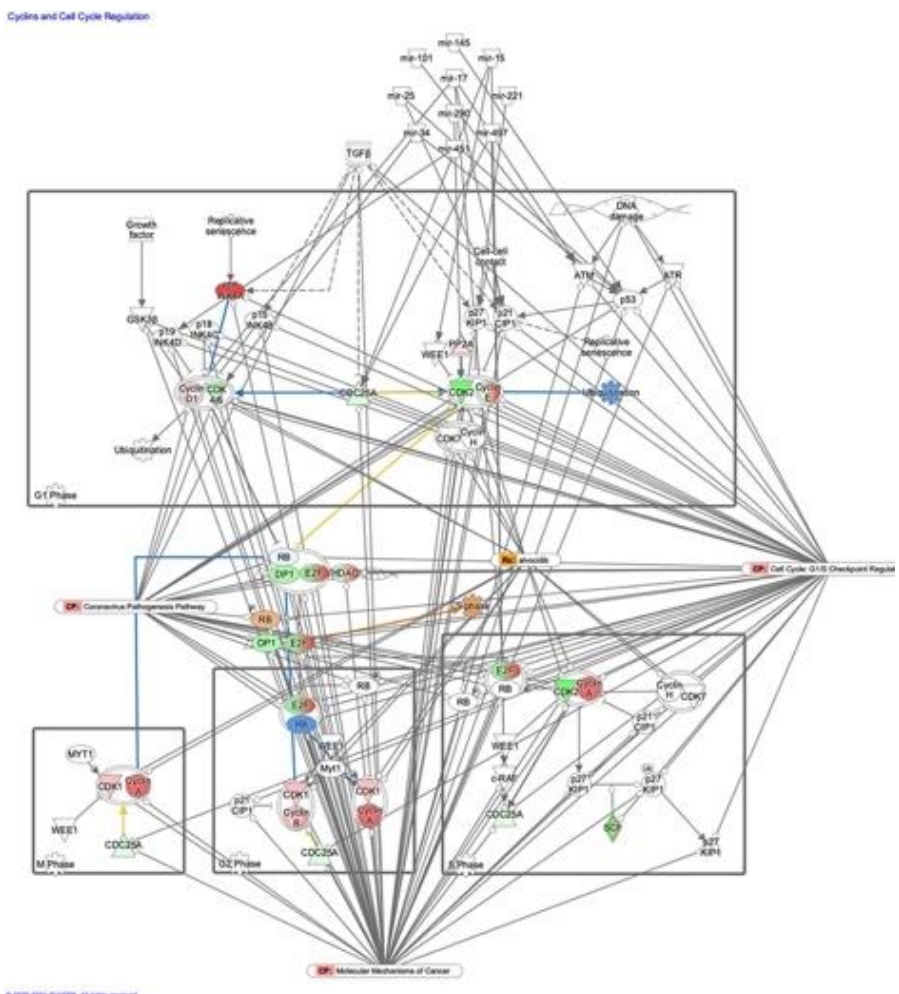

(b)

Figure 4. Cyclins and cell cycle regulation was activated in intestinal-type GC. (a) Gene expression and pathway activity prediction in diffuse-type GC are shown; (b) Gene expression and pathway activity prediction in intestinal-type GC are shown. The genes of which expression was altered in diffuse- and intestinal-type GC are shown in pink (up-regulated) or green (down-regulated). Predicted activation or inhibition is shown in orange or blue, respectively.

Table 4. Direct relationships of miRNAs and targeted molecules in Cyclins and cell cycle regulation.

\begin{tabular}{|c|c|}
\hline From Molecule(s) & To Molecule(s) \\
\hline $\operatorname{mir}-10$ & ATM \\
\hline mir-145 & p53 \\
\hline \multirow{3}{*}{$\operatorname{mir}-15$} & CDC25A \\
\hline & WEE1 \\
\hline & c-RAF \\
\hline \multirow{4}{*}{ mir-17 } & ATM \\
\hline & CyclinD1 \\
\hline & $\mathrm{RB}$ \\
\hline & p21CIP1 \\
\hline mir-221 & p27KIP1 \\
\hline \multirow[t]{2}{*}{ mir-25 } & p21CIP1 \\
\hline & p53 \\
\hline mir-290 & CDK2 \\
\hline \multirow[t]{2}{*}{ mir-34 } & $\mathrm{CDK} 4 / 6$ \\
\hline & p53 \\
\hline mir-451 & p19INK4D \\
\hline
\end{tabular}




$\frac{\text { CDC25A }}{\text { c-RAF }}$

3.2.4. Role of BRCA1 in DNA damage response pathway was activated in intestinal-type GC

Molecule activity predictor in IPA predicted the activation of Role of BRCA1 in DNA damage response pathway in intestinal-type GC (Figure 5). Role of BRCA1 in DNA damage response pathway was identified as the most significant canonical pathway with $p$ value of $6.6 \times 10^{-12}$. Gene expression of BRCA1 which is associated with $\mathrm{G}_{1 / S}$ transition has increased in intestinal-type GC. BRCA1 codes a 190kD nuclear phosphorylation protein that maintains genomic stability and functions as a tumor suppressor. It is interesting that p53 and c21CIP1 are activate in intestinal-type GC in the Role of BRCA1 in DNA damage response pathway. BRCA1 may be involved in the activation of p53. Analysis on direct relationships of RNA-RNA interactions: microRNA targeting in Role of BRCA1 in DNA damage response pathway revealed the relationships between miRNAs and the targeted molecules (Figure 5, Table 5).

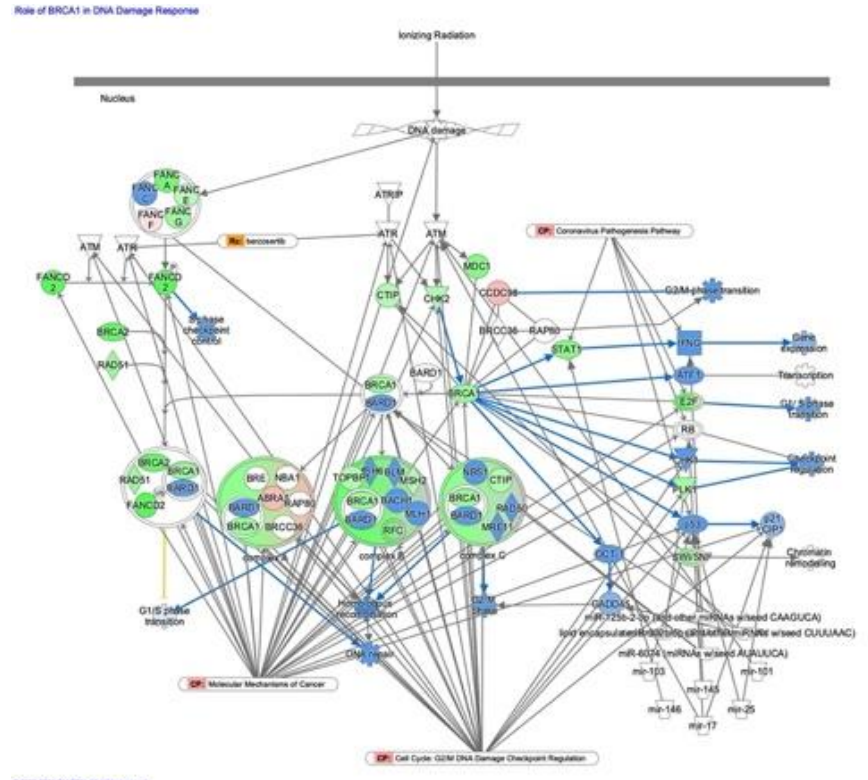

(a)

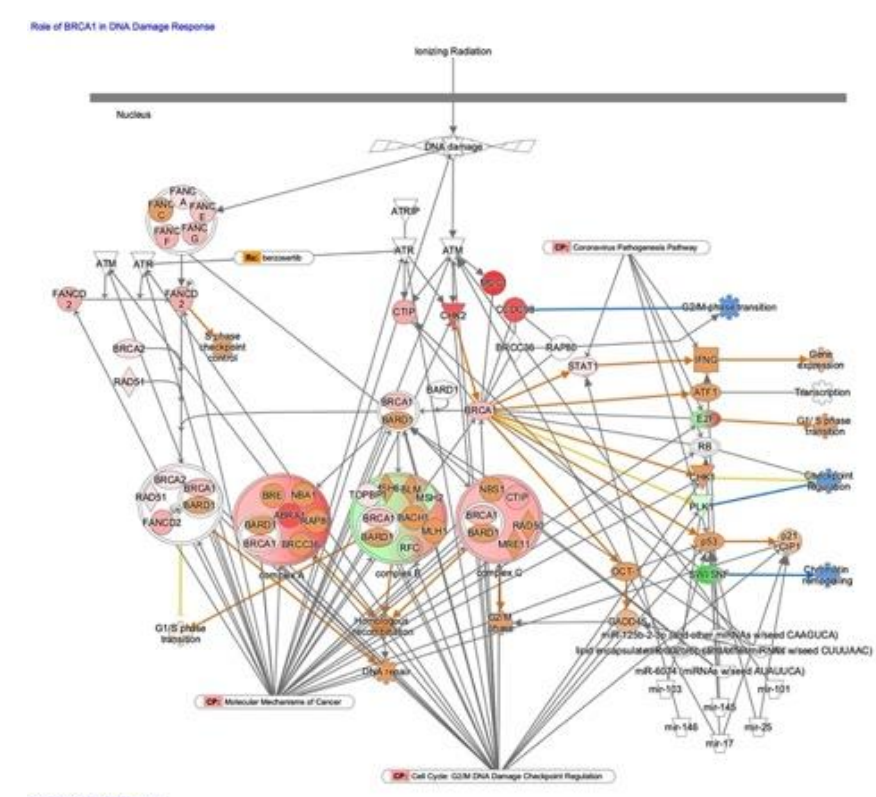

(b)

Figure 5. Role of BRCA1 in DNA damage response was activated in intestinal-type GC. (a) Gene expression and pathway activity prediction in diffuse-type GC are shown; (b) Gene expression and pathway activity prediction in intestinaltype GC are shown. The genes of which expression was altered in diffuse- and intestinal-type GC are shown in pink (upregulated) or green (down-regulated). Predicted activation or inhibition is shown in orange or blue, respectively.

Table 5. Direct relationships of miRNAs and targeted molecules in Role of BRCA1 in DNA damage response.

\begin{tabular}{|c|c|}
\hline From Molecule(s) & To Molecule(s) \\
\hline miR-125b-2-3p (and other miRNAs w/seed CAAGUCA) & $\mathrm{p} 53$ \\
\hline \multirow{3}{*}{ miR-302b-5p (and other miRNAs w/seed CUUUAAC) } & BARD1 \\
\hline & CTIP \\
\hline & GADD45 \\
\hline \multirow{3}{*}{ miR-6074 (miRNAs w/seed AUAUUCA) } & FANCF \\
\hline & IFNG \\
\hline & NBS1 \\
\hline mir-101 & ATM \\
\hline mir-103 & p53 \\
\hline
\end{tabular}




\begin{tabular}{ccc}
\hline mir-145 & p53 \\
\hline mir-146 & STAT1 \\
\cline { 2 - 2 } & $\operatorname{mir}-17$ & $\mathrm{ATM}$ \\
\cline { 2 - 2 } & & $\mathrm{RB}$ \\
\hline $\operatorname{mir}-25$ & $\mathrm{p} 21 \mathrm{p} 21 \mathrm{CIP} 1$ \\
\hline
\end{tabular}

3.2.5. Cell cycle: G2/M DNA damage checkpoint regulation pathway in diffuse- and intestinal- type GC

Cell cycle: G2/M DNA damage checkpoint regulation pathway was identified as related canonical pathway in diffuse- and intestinal- type GC (Figure 6). Analysis on direct relationships of RNA-RNA interaction in Cell cycle: $\mathrm{G}_{2} / \mathrm{M}$ DNA damage checkpoint regulation pathway revealed the non-coding RNAs including 9 miRNAs and a biologic drug (lipid encapsulated anti-PLK1 siRNA TKM-080301) (Table 6).

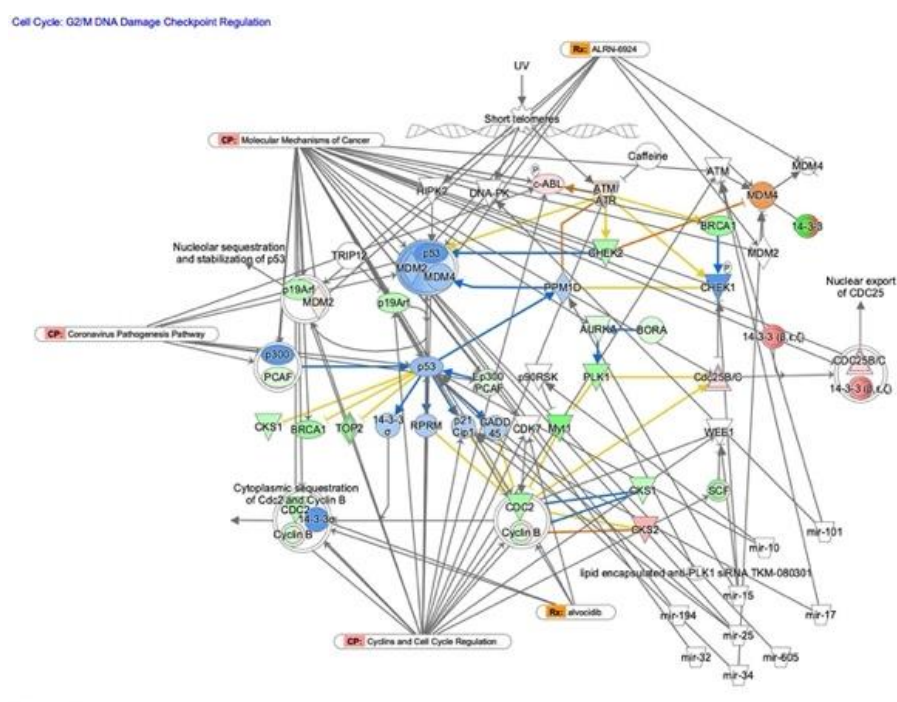

(a)

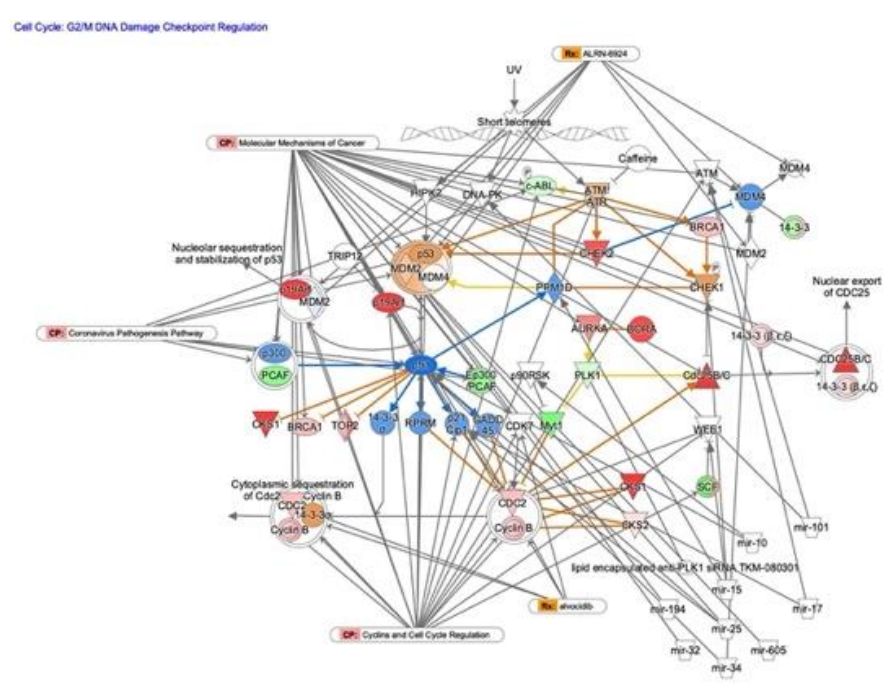

(b)

Figure 6. Cell cycle: $\mathrm{G}_{2} / \mathrm{M}$ DNA damage checkpoint regulation pathway in diffuse- and intestinal- type GC. (a) Gene expression and pathway activity prediction in diffuse-type GC are shown; (b) Gene expression and pathway activity prediction in intestinal-type GC are shown. The genes of which expression was altered in diffuse- and intestinal-type GC are shown in pink (up-regulated) or green (down-regulated). Predicted activation or inhibition is shown in orange or blue, respectively.

Table 6. Direct relationships of miRNAs and targeted molecules in Cell cycle: G2/M DNA damage checkpoint regulation pathway.

\begin{tabular}{cccc}
\hline Symbol & Entrez Gene Name & Location & Family \\
\hline lipid encapsulated anti-PLK1 siRNA TKM-080301 & & Other & biologic drug \\
\hline mir-10 & microRNA 99a & & \\
\hline mir-101 & microRNA 101-1 & & \\
\hline mir-15 & microRNA 15a & \\
\hline mir-17 & Cytoplasm & microRNA \\
\hline mir-194 & microRNA 194-1 & & \\
\hline mir-25 & microRNA 25 \\
\hline mir-32 & microRNA 32 \\
\hline mir-34 & microRNA 34a \\
\hline mir-605 & microRNA 605 & \\
\hline
\end{tabular}




\section{Discussion}

From the results of network analysis in diffuse- and intestinal-type GC, several canonical pathways related to cell cycle regulation including "Cell cycle control of chromosomal replication", "Cell cycle: G1/S checkpoint regulation", "Cyclins and cell cycle regulation", and "Cell cycle: G2/M DNA damage checkpoint regulation" have been identified to alter. A previous study showed that $\mathrm{G}_{1} / \mathrm{S}$ arrest induces EMT by ribosome biogenesis [14]. This finding of $\mathrm{G}_{1} / \mathrm{S}$ arrest in EMT may be associated with the finding in the current study in terms of activating the "Cell cycle: $\mathrm{G}_{1} / \mathrm{S}$ checkpoint regulation" pathway in diffuse-type GC. SMAD4, which was activated in diffuse-type GC, is involved in EMT [15]. The silencing of SMAD4 reversed EMT in hepatocytes [16]. The several miRNAs have been found to have direct relationships in pathways related to cell cycle regulation. In "Cell cycle: G1/S checkpoint regulation", mir-10 (microRNA 99a), mir-17 (microRNA 17), mir-19 (microRNA 19a), mir-194 (microRNA 194-1), mir-224 (microRNA 224), mir-25 (microRNA 25), mir-34 (microRNA 34a), mir-451 (microRNA 451a), mir-605 (microRNA 605), and MYC-targeting siRNA DCR-MYC were identified as molecules having the direct relationships (RNA-RNA interactions). It has been previously revealed that miR-17/20 cluster, which is transcriptionally regulated by MYC, E2F, and cyclin D1, and miR-34, is a direct transcriptional target of p53 and regulates cell cycle [17]. The cell cycle regulation of miRNAs can be involved in cancer. Small nucleolar RNA host gene 7 (SNHG7), an oncogenic long non-coding RNA, promotes cell migration via miR-34a-Snail-EMT axis in gastric cancer [18]. The involvement of miR-34 in EMT and GC is consistent with the results showing the direct relationship between miR-34 and "Cell cycle: G1/S checkpoint regulation" pathway, which is activated in diffuse-type GC. Another study suggested that miRNA-33a inhibited EMT and metastasis of GC via the Snail/Slug pathway, which may be related to the miRNA regulation in EMT in intestinal-type GC [19]. MYC-targeting siRNA, a biologic drug targeting MYC, has been identified to have direct relationship in "Cell cycle: $\mathrm{G}_{1} / \mathrm{S}$ checkpoint regulation". It has been shown before that the silencing of Aurora kinase B, which is up-regulated in GC, decreased the expression of MYC, arrested the cell cycle in $\mathrm{G}_{2} / \mathrm{M}$ phase, and inhibited migration of GC [20]. The MYC-targeting siRNA might also be involved in the regulation of cell cycle and EMT.

The current study highlights the cell cycle regulation and non-coding RNA regulation in diffuse- and intestinal-type GC. A long non-coding RNA LINC00460 induced EMT and cell proliferation and metastasis in head and neck squamous cell carcinoma via translocation of peroxiredoxin-1 into the cell nucleus [21]. The non-coding RNAs, such as microRNAs, have various roles in biological and pathological processes, including cell-cycle, proliferation, EMT, and drug resistance [22]. The investigation in RNA regulation and EMT in the point of view of anti-cancer drug resistance and identification of therapeutic targets would be the future research direction.

\section{Conclusions}

The several canonical pathways have been found to be altered in diffuse- and intestinal-type GC. Canonical pathway on "Cell cycle: G1/S checkpoint regulation" was activated in diffuse-type GC, and "Cell cycle control of chromosomal replication" pathway and "Cyclins and cell cycle regulation" pathway was activated in intestinal-type GC. Canonical pathway related to "Role of BRCA1 in DNA damage response" was activated in intestinal-type GC compared to diffuse-type GC. Some molecules such as SMAD4 in the pathway of "Cell cycle: G1/S checkpoint regulation" are involved in regulation of EMT. Cell cycle regulation may also be altered in EMT conditions in diffuse-type GC. The findings of the study where the activation states in the pathways related to cell cycle regulation alter in diffuse- and intestinal-type GC would highlight the significance of the cell 
cycle regulation in EMT. Precise mechanism where compartment molecules in cell cycle pathways regulate EMT and CSCs would be future investigation.

Author Contributions: Conceptualization, S.T. and H.S.; methodology, S.T.; software, S.T.; formal analysis, S.T.; investigation, S.T.; data curation, S.T., K.A. and H.S.; writing - original draft preparation, S.T.; writing - review and editing, S.T., S.Q. and H.C.; visualization, S.T.; supervision, S.T. and A.H.; project administration, S.T., K.A., H.Y. and H.S.; funding acquisition, S.T., S.Q., R.O. and A.H. All authors have read and agreed to the published version of the manuscript.

Funding: This work was supported by Japan Agency for Medical Research and Development (AMED), Grant Numbers JP21mk0101216 (S.T.), JP20ak0101093 (S.T., R.O. and A.H.) and JP20mk0101163 (RO), and Strategic International Collaborative Research Program, Grant Number JP20jm0210059 (S.T. and S.Q.), Ministry of Health, Labour, and Welfare (MHLW), Grant Number H30-KAGAKU-IPPAN-002 (S.T. and R.O.), and JSPS KAKENHI Grant Number 21K12133 (S.T. and R.O.).

Data Availability Statement: In this research, the RefSeq data of diffuse- and intestinal-type GC publicly available in The Cancer Genome Atlas (TCGA) of The cBioPortal for Cancer Genomics database [9-11] in National Cancer Institute (NCI) Genomic Data Commons (GDC) Data Portal was analyzed [12]. From the publicly available data of stomach adenocarcinoma in TCGA (NCI, USA: https://www.cancer.gov/about-nci/organization/ccg/research/structural-genomics/tcga) [10], intestinal- and diffuse-type GC data, which are noted as chromosomal instability (CIN) $(n=223)$ and genomically stable (GS) $(n=50)$, respectively, in TCGA Research Network publication, were compared [11].

Acknowledgments: The authors would like to thank all colleagues for their support.

Conflicts of Interest: The authors declare no conflict of interest. The funders had no role in the design of the study; in the collection, analyses, or interpretation of data; in the writing of the manuscript, or in the decision to publish the results.

\section{References}

1. Shibue, T.; Weinberg, R.A. EMT, CSCs, and drug resistance: the mechanistic link and clinical implications. Nat Rev Clin Oncol 2017, 14, 611-629, doi:10.1038/nrclinonc.2017.44.

2. Tanabe, S.; Quader, S.; Ono, R.; Cabral, H.; Aoyagi, K.; Hirose, A.; Yokozaki, H.; Sasaki, H. Molecular Network Profiling in Intestinal- and Diffuse-Type Gastric Cancer. Cancers (Basel) 2020, 12, 3833, doi:10.3390/cancers12123833.

3. Tanabe, S.; Quader, S.; Cabral, H.; Ono, R. Interplay of EMT and CSC in Cancer and the Potential Therapeutic Strategies. Front Pharmacol 2020, 11, 904, doi:10.3389/fphar.2020.00904.

4. Xu, H.; Chen, R.; Shen, Q.; Yang, D.; Peng, H.; Tong, J.; Fu, Q. Overexpression of Circular RNA circ_0013587 Reverses Erlotinib Resistance in Pancreatic Cancer Cells Through Regulating the miR-1227/E-Cadherin Pathway. Front Oncol 2021, 11, 754146, doi:10.3389/fonc.2021.754146.

5. Chen, S.; Wu, H.; Zhu, L.; Jiang, M.; Wei, S.; Luo, J.; Liu, A. MiR-199b-5p Promotes Gastric Cancer Progression by Regulating HHIP Expression. Front Oncol 2021, 11, 728393, doi:10.3389/fonc.2021.728393.

6. $\mathrm{Xu}, \mathrm{J}$.; Wang, X.; Wang, W.; Zhang, L.; Huang, P. Candidate oncogene circularNOP10 mediates gastric cancer progression by regulating miR-204/SIRT1 pathway. J Gastrointest Oncol 2021, 12, 1428-1443, doi:10.21037/jgo-21-422.

7. Tanabe, S.; Aoyagi, K.; Yokozaki, H.; Sasaki, H. Gene expression signatures for identifying diffuse-type gastric cancer associated with epithelial-mesenchymal transition. Int J Oncol 2014, 44, 1955-1970, doi:10.3892/ijo.2014.2387.

8. Tanabe, S.; Kawabata, T.; Aoyagi, K.; Yokozaki, H.; Sasaki, H. Gene expression and pathway analysis of CTNNB1 in cancer and stem cells. World J Stem Cells 2016, 8, 384-395, doi:10.4252/wjsc.v8.i11.384. 
9. Cerami, E.; Gao, J.; Dogrusoz, U.; Gross, B.E.; Sumer, S.O.; Aksoy, B.A.; Jacobsen, A.; Byrne, C.J.; Heuer, M.L.; Larsson, E., et al. The cBio Cancer Genomics Portal: An Open Platform for Exploring Multidimensional Cancer Genomics Data. Cancer Discovery 2012, 2, 401, doi:10.1158/2159-8290.CD-12-0095.

10. Gao, J.; Aksoy, B.A.; Dogrusoz, U.; Dresdner, G.; Gross, B.; Sumer, S.O.; Sun, Y.; Jacobsen, A.; Sinha, R.; Larsson, E., et al. Integrative analysis of complex cancer genomics and clinical profiles using the cBioPortal. Sci Signal 2013, 6, pl1, doi:10.1126/scisignal.2004088.

11. Bass, A.J.; Thorsson, V.; Shmulevich, I.; Reynolds, S.M.; Miller, M.; Bernard, B.; Hinoue, T.; Laird, P.W.; Curtis, C.; Shen, H., et al. Comprehensive molecular characterization of gastric adenocarcinoma. Nature 2014, 513, 202-209, doi:10.1038/nature13480.

12. Grossman, R.L.; Heath, A.P.; Ferretti, V.; Varmus, H.E.; Lowy, D.R.; Kibbe, W.A.; Staudt, L.M. Toward a Shared Vision for Cancer Genomic Data. N Engl J Med 2016, 375, 1109-1112, doi:10.1056/NEJMp1607591.

13. Krämer, A.; Green, J.; Pollard, J., Jr.; Tugendreich, S. Causal analysis approaches in Ingenuity Pathway Analysis. Bioinformatics 2014, 30, 523-530, doi:10.1093/bioinformatics/btt703.

14. Prakash, V.; Carson, B.B.; Feenstra, J.M.; Dass, R.A.; Sekyrova, P.; Hoshino, A.; Petersen, J.; Guo, Y.; Parks, M.M.; Kurylo, C.M., et al. Ribosome biogenesis during cell cycle arrest fuels EMT in development and disease. Nat Commun 2019, 10, 2110, doi:10.1038/s41467-019-10100-8.

15. Chen, B.; Ma, J.; Li, C.; Wang, Y. Long noncoding RNA KCNQ1OT1 promotes proliferation and epithelial-mesenchymal transition by regulation of SMAD4 expression in lens epithelial cells. Mol Med Rep 2018, 18, 16-24, doi:10.3892/mmr.2018.8987.

16. Kaimori, A.; Potter, J.; Kaimori, J.Y.; Wang, C.; Mezey, E.; Koteish, A. Transforming growth factor-beta1 induces an epithelial-to-mesenchymal transition state in mouse hepatocytes in vitro. J Biol Chem 2007, 282, 22089-22101, doi:10.1074/jbc.M700998200.

17. Yu, Z.; Baserga, R.; Chen, L.; Wang, C.; Lisanti, M.P.; Pestell, R.G. microRNA, cell cycle, and human breast cancer. Am J Pathol 2010, 176, 1058-1064, doi:10.2353/ajpath.2010.090664.

18. Zhang, Y.; Yuan, Y.; Zhang, Y.; Cheng, L.; Zhou, X.; Chen, K. SNHG7 accelerates cell migration and invasion through regulating miR-34a-Snail-EMT axis in gastric cancer. Cell Cycle 2020, 19, 142-152, doi:10.1080/15384101.2019.1699753.

19. Chen, D.D.; Cheng, J.T.; Chandoo, A.; Sun, X.W.; Zhang, L.; Lu, M.D.; Sun, W.J.; Huang, Y.P. microRNA-33a prevents epithelial-mesenchymal transition, invasion, and metastasis of gastric cancer cells through the Snail/Slug pathway. Am $J$ Physiol Gastrointest Liver Physiol 2019, 317, G147-g160, doi:10.1152/ajpgi.00284.2018.

20. Wang, Z.; Yu, Z.; Wang, G.H.; Zhou, Y.M.; Deng, J.P.; Feng, Y.; Chen, J.Q.; Tian, L. AURKB Promotes the Metastasis of Gastric Cancer, Possibly by Inducing EMT. Cancer Manag Res 2020, 12, 6947-6958, doi:10.2147/cmar.S254250.

21. Jiang, Y.; Cao, W.; Wu, K.; Qin, X.; Wang, X.; Li, Y.; Yu, B.; Zhang, Z.; Wang, X.; Yan, M., et al. LncRNA LINC00460 promotes EMT in head and neck squamous cell carcinoma by facilitating peroxiredoxin-1 into the nucleus. J Exp Clin Cancer Res 2019, 38, 365, doi:10.1186/s13046-019-1364-z.

Wang, J.Y.; Yang, Y.; Ma, Y.; Wang, F.; Xue, A.; Zhu, J.; Yang, H.; Chen, Q.; Chen, M.; Ye, L., et al. Potential regulatory role of lncRNA-miRNA-mRNA axis in osteosarcoma. Biomed Pharmacother 2020, 121, 109627, doi:10.1016/j.biopha.2019.109627. 